\title{
Inmunoterapia con veneno de himenópteros: presentación de diez casos clínicos
}

\author{
María Antonieta G uzmán M ${ }^{1}$, Jessica Salinas L1, Paola \\ Toche $P^{1}$, María Angélica Marinovic $M^{1}$, Ana María \\ Gallardo $0^{2}$ \\ Immunotherapy against \\ hymenoptera venom. Report of ten \\ patients
}

Background: Immunotherapy can be used to treat allergic reactions to insect stings, specially bees and wasps. Aim: To report the experience with immunotherapy with aqueous extracts of hymenoptera venoms (bees and wasps). Material and methods: Ten patients aged 6 to 58 years were treated in an allergy center of a University Clinical Hospital. The medical indication for this treatment was, in all patients, anaphylactic reactions after bymenoptera stings. Immunotherapy was carried out using standardized vaccines (Aqueous extracts Venomvac LETI, Spain), applied in a traditional protocol, with subcutaneous injections. This protocol had two phases: a buildup phase (between weeks 1 and 13) and a monthly maintenance phase, from the 13th week. The monthly maintenance dose was $100 \mu \mathrm{g}$ of hymenoptera specific venom extract. Results: Six patients had adverse reactions of different severity, during the treatment protocols and all had a good response to immediate therapeutic measures. After these events, they followed the protocol without problems. Two patients, treated with bee vaccines, suffered an accidental bee sting during the maintenance phase and they developed only local reactions. Conclusions: The lack of adverse reactions to bee stings in these two patients indicates the acquisition of clinical tolerance (Rev Méd Chile 2007; 135: 1566-71).

(Key words: Desensitization, immunologic; Hymenoptera; Hypersensitivity; Venoms)

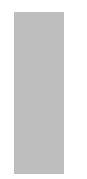

\begin{abstract}
Recibido el 27 de junio, 2006. Aceptado el 16 de abril, 2007. Apoyo financiero: No existió fuente de apoyo financiero.

${ }^{1}$ Centro de Alergias, Sección Inmunología y Alergología, Departamento de Medicina, Hospital Clínico Universidad de Chile. ${ }^{2}$ Programa de Especialistas en Inmunología Clínica, Escuela de Postgrado, Universidad de Chile. Santiago de Chile.
\end{abstract}

$\mathrm{L}$ a inmunoterapia (IT) consiste en administrar cantidades gradualmente crecientes del alergeno que ha provocado síntomas de hipersensibili-

Correspondencia a: Dra. María Antonieta Guzmán M. Hospital Clínico Universidad de Chile. Santos Dumont 999, $5^{\circ}$ piso sector E. Sección Inmunología y Alergología. Código postal 8380450, Santiago. Tel: 56-2-9788567. Fax: 56-27375916. E mail: mguzman@redclinicauchile.cl dad inmediata, con el fin de lograr tolerancia clínica ante una nueva exposición al alérgeno ${ }^{1}$. Actualmente constituye el único tratamiento etiológico en el manejo de las patologías alérgicas.

Entre sus indicaciones, reconocidas internacionalmente ${ }^{1}$, destacan las reacciones anafilácticas por picadura de insectos himenópteros (abejas, avispas), donde la inmunoterapia tiene su mayor efectividad. 
Se presentan 10 casos clínicos de pacientes tratados con extractos comerciales acuosos específicos de veneno de himenópteros Venomvac, LETI, España, en el Centro de Alergias del Hospital Clínico de la Universidad de Chile. Estos extractos de inmunoterapia son comercializados en nuestro país desde hace 5 años.

\section{Casos CLínicos}

Presentamos una serie de 10 pacientes, quienes consultaron por reacciones alérgicas generalizadas e inmediatas por picaduras de abeja (9 casos) o avispa (1 caso), de diversa severidad, como se muestra en la Tabla 1. En todos se demostró in

Tabla 1. C aracterización de pacientes en IT con veneno de himenópteros

\begin{tabular}{|c|c|c|c|c|c|c|}
\hline $\begin{array}{c}\mathrm{N}^{\circ} \mathrm{de} \\
\text { paciente e } \\
\text { insecto } \\
\text { causal }\end{array}$ & $\begin{array}{l}\text { Sexo y } \\
\text { edad } \\
\text { (años) al } \\
\text { inicio de } \\
\text { la IT }\end{array}$ & $\begin{array}{l}\text { Región de } \\
\text { origen }\end{array}$ & $\begin{array}{l}\text { Valor IgE } \\
\text { específica } \\
\text { y método }\end{array}$ & $\begin{array}{l}\text { Tipo de } \\
\text { reacción } \\
\text { sistémica* }\end{array}$ & Inicio IT & $\begin{array}{c}\text { Re-exposición } \\
\text { durante IT }\end{array}$ \\
\hline $\begin{array}{c}1 \\
\text { Abeja }\end{array}$ & M, 58 & VII (rural) & $\begin{array}{c}\text { Clase VI } \\
(>100 \mathrm{UI} / \mathrm{ml})\end{array}$ & Grado 3 & $\begin{array}{l}\text { Marzo } \\
2003\end{array}$ & $\begin{array}{l}\text { Sí: Reacción } \\
\text { local menor }\end{array}$ \\
\hline $\begin{array}{c}2 \\
\text { Abeja }\end{array}$ & F, 9 & V (rural) & $\begin{array}{c}\text { Clase IV } \\
(26,6 \mathrm{UI} / \mathrm{ml})\end{array}$ & Grado 2 & $\begin{array}{l}\text { Abril } \\
2003\end{array}$ & $\begin{array}{l}\text { Sí: Reacción } \\
\text { local menor }\end{array}$ \\
\hline $\begin{array}{c}3 \\
\text { Abeja }\end{array}$ & M, 6 & VII (urbano) & $\begin{array}{c}\text { Clase II } \\
(2,8 \mathrm{UI} / \mathrm{ml})\end{array}$ & Grado 3 & $\begin{array}{l}\text { Marzo } \\
2004\end{array}$ & No \\
\hline $\begin{array}{c}4 \\
\text { Abeja }\end{array}$ & M, 54 & $\begin{array}{l}\text { Metropolitana } \\
\text { (rural) }\end{array}$ & $\begin{array}{l}\text { Clase V } \\
(80 \mathrm{UI} / \mathrm{ml})\end{array}$ & Grado 3 & $\begin{array}{c}\text { Febrero } \\
2005\end{array}$ & No \\
\hline $\begin{array}{c}5 \\
\text { Abeja }\end{array}$ & F, 21 & $\begin{array}{l}\text { Metropolitana } \\
\text { (urbano) }\end{array}$ & Clase II $^{* *}$ & Grado 2 & $\begin{array}{l}\text { Marzo } \\
2005\end{array}$ & No \\
\hline $\begin{array}{c}6 \\
\text { Abeja }\end{array}$ & M, 7 & $\begin{array}{l}\text { Metropolitana } \\
\text { (urbano) }\end{array}$ & $\begin{array}{c}\text { Clase VI } \\
(>100 \mathrm{UI} / \mathrm{ml})\end{array}$ & Grado 3 & $\begin{array}{l}\text { Abril } \\
2005\end{array}$ & No \\
\hline $\begin{array}{c}7 \\
\text { Abeja }\end{array}$ & F, 17 & $\begin{array}{l}\text { Metropolitana } \\
\quad \text { (rural) }\end{array}$ & $\begin{array}{c}\text { Clase III } \\
(14,8 \mathrm{UI} / \mathrm{ml})\end{array}$ & Grado 2 & $\begin{array}{l}\text { Agosto } \\
2005\end{array}$ & No \\
\hline $\begin{array}{c}8 \\
\text { Abeja }\end{array}$ & M, 8 & $\begin{array}{l}\text { Metropolitana } \\
\quad \text { (rural) }\end{array}$ & $\begin{array}{c}\text { Clase V } \\
(90,3 \mathrm{UI} / \mathrm{ml}\end{array}$ & Grado 3 & $\begin{array}{l}\text { Agosto } \\
2005\end{array}$ & No \\
\hline $\begin{array}{c}9 \\
\text { Abeja }\end{array}$ & F, 31 & $\begin{array}{l}\text { VII Región } \\
\text { (urbano) }\end{array}$ & $\begin{array}{c}\text { Clase VI } \\
(>100 \mathrm{UI} / \mathrm{ml})\end{array}$ & Grado 3 & $\begin{array}{r}\text { Mayo } \\
2006\end{array}$ & No \\
\hline $\begin{array}{c}10 \\
\text { Avispa }\end{array}$ & $\mathrm{F}, 11$ & VII (rural) & $\begin{array}{c}\text { Clase II } \\
(1,4 \mathrm{UI} / \mathrm{ml})\end{array}$ & Grado 3 & $\begin{array}{l}\text { Junio } \\
2006\end{array}$ & No \\
\hline
\end{tabular}

F: Femenino, M: Masculino

* Según Clasificación de Mueller UR. ${ }^{7}$

Grado I: Urticaria generalizada (incluye edema periorbitario), prurito, malestar y ansiedad.

Grado II: Angioedema o dos o más de los siguientes: sensación de opresión torácica, ocupación faríngea, náuseas, vómitos, diarrea, dolor abdominal, mareos.

Grado III: Disnea, sibilancias o estridor o dos o más de los siguientes: disfagia, disartria, ronquera, debilidad, confusión, sensación de desastre inminente.

Grado IV: Hipotensión, colapso, pérdida de conciencia, incontinencia urinaria o fecal, cianosis.

** Esta es la paciente en que se midió IgE específicas por Unicap, y no se entregó información respecto de las Unidades. 
vitro la presencia de IgE específicas contra venenos (de clase II a clase VI), por lo cual no fue necesario efectuar pruebas cutáneas de hipersensibilidad inmediata con fines diagnósticos. La medición de IgE específicas se realizó por enzimoinmoensayos, en 9 pacientes por técnica de Inmulite (DPC, Los Ángeles, USA), y en una paciente por método de UniCap (Pharmacia, Upsala, Suecia).

Los 10 pacientes recordaban exposición previa a picaduras del insecto causal, lo que explica su sensibilización alérgica. Algunos habían sido picados previamente por abejas y avispas, lo que es potencialmente importante, porque entre los alergenos de ambos venenos existen grados variables de reactividad cruzada $^{2-4}$.

Todos nuestros pacientes reconocían claramente el insecto causal de las reacciones y en quienes habían experimentado reacciones anafilácticas posterior a la picadura de abejas, era claro el antecedente de desprendimiento del aguijón en el sitio de la picadura.

Tabla 2. Reacciones adversas durante IT con extracto acuoso de veneno de himenópteros
(Venomvac, LET I, España)

\begin{tabular}{|c|c|c|c|c|}
\hline Paciente $\mathrm{n}^{\circ}$ & Tipo de reacción & $\begin{array}{c}\text { Dosis } \\
\text { extracto }\end{array}$ & $\begin{array}{c}\text { Mes o } \\
\text { semana de } \\
\text { administración }\end{array}$ & Tratamiento \\
\hline \multirow[t]{2}{*}{2} & Induración local & $10 \mu \mathrm{g}$ & 7a semana & $\begin{array}{l}\text { Antihistamínico } \\
\text { oral, compresa fría }\end{array}$ \\
\hline & $\begin{array}{c}\text { Broncoespasmo } \\
\text { y urticaria }\end{array}$ & $100 \mu \mathrm{g}$ & $17^{\circ}$ mes & $\begin{array}{c}\text { Broncodilatador } \\
\text { aerosol, } \\
\text { Antihistamínico y } \\
\text { Corticoide I.M. }\end{array}$ \\
\hline \multirow[t]{3}{*}{3} & $\begin{array}{l}\text { Induración local } \\
\text { retardada }(18 \text { h) }\end{array}$ & $10 \mu \mathrm{g}$ & 7a semana & $\begin{array}{l}\text { Antihistamínico } \\
\text { oral, compresa fría }\end{array}$ \\
\hline & $\begin{array}{c}\text { Broncoespasmo } \\
\text { (sibilancias } \\
\text { aisladas) }\end{array}$ & $100 \mu \mathrm{g}$ & $5^{\circ}, 8^{\circ}$ y $9^{\circ}$ mes & $\begin{array}{c}\text { Broncodilatador } \\
\text { aerosol }\end{array}$ \\
\hline & $\begin{array}{c}\text { Reacción local } \\
\text { mayor }\end{array}$ & $100 \mu \mathrm{g}$ & $16^{\circ} \mathrm{mes}$ & $\begin{array}{c}\text { Antihistamínico oral, } \\
\text { Corticoide I.M. }\end{array}$ \\
\hline 4 & $\begin{array}{c}\text { Reacción local } \\
\text { mayor }\end{array}$ & $100 \mu \mathrm{g}$ & $13^{\mathrm{a}}$ semana & $\begin{array}{c}\text { Corticoide y } \\
\text { antihistamínico } \\
\text { orales }\end{array}$ \\
\hline 6 & Induración local & $20 \mu \mathrm{g}$ & $8^{\underline{a}}$ semana & $\begin{array}{c}\text { Antihistamínico } \\
\text { oral }\end{array}$ \\
\hline 7 & $\begin{array}{l}\text { Sensación } \\
\text { de ocupación } \\
\text { faríngea, examen } \\
\text { físico negativo }\end{array}$ & $100 \mu \mathrm{g}$ & $13^{\underline{a}}$ semana & $\begin{array}{c}\text { Antihistamínico } \\
\text { oral }\end{array}$ \\
\hline 8 & $\begin{array}{l}\text { Prurito } \\
\text { palmoplantar, } \\
\text { reacción local } \\
\text { mayor }\end{array}$ & $100 \mu \mathrm{g}$ & $13^{\mathrm{a}}$ semana & $\begin{array}{l}\text { Antihistamínico } \\
\text { I.M., Corticoide } \\
\text { oral }\end{array}$ \\
\hline
\end{tabular}

Nota: Dosis de mantención alcanzada a la $13^{\text {a }}$ semana, de allí en adelante, se trata de dosis mensuales (fase de mantención) 
Respecto a exposición laboral, el paciente $\mathrm{n}^{\circ} 1$ es un apicultor de la VII Región, expuesto a múltiples picaduras desde su juventud y presenta un pénfigo cutáneo en tratamiento con prednisona en dosis bajas y dapsona $50 \mathrm{mg} /$ día, desde hace 6 años, lo que no previno la ocurrencia de reacciones anafilácticas graves ante picaduras de abejas, previas a la IT.

Nuestros pacientes están en tratamiento de IT específica con extracto acuoso Venomvac (LETI, España), todos ellos en fase de mantención, recibiendo inyecciones mensuales subcutáneas equivalentes a $100 \mu \mathrm{g}$ de veneno.

El esquema de IT utilizado consiste en una pauta tradicional, vía subcutánea, partiendo con $0,01 \mu \mathrm{g}$ del veneno específico, aumentando gradualmente la dosis en 13 semanas (fase de incremento), hasta alcanzar la dosis de mantención de $100 \mu \mathrm{g}$, que se administra en forma mensual.

Este procedimiento se efectúa ambulatoriamente en nuestro centro hospitalario, donde contamos con los recursos adecuados para el tratamiento de eventuales reacciones anafilácticas, previa firma del consentimiento informado por el paciente o sus padres (en caso de menores de edad). Los pacientes son controlados por médico hasta 1 hora después de la colocación de la dosis de IT.

Hasta marzo de 2007, estos pacientes siguen la pauta mencionada, en promedio, hace 26,9 meses (9-49 meses) y han presentado una buena tolerancia al tratamiento.

Respecto a los efectos adversos, en nuestra serie se presentaron en 6 pacientes (Tabla 2) e incluyeron reacciones locales, locales mayores y sistémicas. Ninguna de estas fue severa ni requirió de hospitalización. La paciente $n^{\circ} 2$ quien presentó broncoespasmo y urticaria alejada del sitio de inyección en relación a una dosis de mantención al $17^{\circ}$ mes de tratamiento, respondió a broncodilatadores en aerosol, antihistamínicos y corticoides parenterales (I.M.) y en la siguiente sesión se colocó la dosis previamente tolerada.

Además, en quienes presentaron reacciones adversas, y dependiendo de su severidad, se indicó profilaxis con antihistamínicos o con corticoides orales, el día previo y el día de la inmunoterapia, con buenos resultados.

Cinco de los seis pacientes que presentaron reacciones adversas, lo hicieron por primera vez con la utilización de diversos volúmenes del frasco más concentrado $(100 \mu \mathrm{g} / \mathrm{ml})$, y en la fase de ascenso (principalmente semanas $7 \underline{a}$ a $13^{\underline{a}}$ ) y en especial, al alcanzar la dosis de mantención de $100 \mu \mathrm{g} / \mathrm{ml}$ (semana 13aㅡ). En el caso de IT con veneno de abeja, esta dosis representa la cantidad inyectada por la picadura de 2 abejas.

Dos de nuestros pacientes $\left(\mathrm{n}^{\circ} 1 \mathrm{y}^{\circ} 2\right)$, han experimentado nuevas picaduras de abeja en forma accidental, presentando sólo reacciones locales, demostrando así la adquisición de tolerancia clínica con la IT.

\section{Discusión}

El tratamiento de una picadura de himenópteros se divide en tratamiento de emergencia e inmunoterapia veneno específica a largo plazo. No es el objetivo de esta revisión analizar en detalle el tratamiento de emergencia de una reacción anafiláctica por picadura de himenópteros, sólo mencionaremos la importancia de retirar inmediatamente el aguijón en picaduras de abeja, sin exprimirlo y la utilización de epinefrina $a^{5,6}$.

La inmunoterapia está indicada solamente en reacciones generalizadas ${ }^{3}$ (urticaria, angioedema, compromiso respiratorio o cardiovascular) después de una picadura de abeja o avispa, con demostración, in vitro (por enzimoinmunoensayos) o in vivo (por pruebas cutáneas) de IgE específicas contra el veneno causal (sin que su magnitud, medida en clases y unidades, sea un factor determinante para decidir la IT).

Junto con la caracterización clínica de una reacción alérgica generalizada dentro de los minutos posteriores a la picadura de un himenóptero, debe identificarse el insecto causal, ya que los pacientes que experimentan estas reacciones tienen indicación de IT con un extracto de veneno específico ${ }^{1,8,9}$. Las características físicas de las abejas (color café y presencia de vellosidad) y el hecho de que se desprenden de su aguijón durante la picadura, contribuyeron a su identificación por los pacientes y sus familiares ${ }^{10,11}$.

La IT con veneno puede causar grandes reacciones locales de días de duración e incluso, reacciones sistémicas ${ }^{2,10}$ en $5 \%$ a $12 \%$ de los pacientes ${ }^{12}$. Estas son más frecuentes durante la fase inicial de incremento de $\operatorname{dosis}^{12}$, lo que 
ocurrió también en nuestra serie, en particular, en las semanas inmediatamente previas a alcanzar la dosis de mantención. Las reacciones locales mayores, ocurren en $25 \%$ de los pacientes en IT, no requiriendo ajustar la dosis ni reducirla, pues las tasas de riesgo de reacciones sistémicas posteriores son bajas $(0,8-1,01 \%)^{13}$. En nuestro caso, solo la paciente $\mathrm{n}^{\circ} 2$, requirió una reducción de dosis a la dosis previamente tolerada, luego de presentar una reacción generalizada bajo IT, pudiendo luego retomar el esquema originalmente planteado. Esta paciente, además, enfatiza que jamás podemos confiarnos en que estas reacciones no se presentarán por el hecho de haber alcanzado la etapa de mantención sin reacciones previas, y por lo tanto, la IT es un procedimiento que siempre va a requerir una vigilancia estricta, por parte de los tratantes.

Respecto a la edad, se acepta efectuar IT desde los 5 años ${ }^{1,3,8,10,14}$, salvo un gran riesgo de exposición que justifique plantearla antes, especialmente si han ocurrido reacciones generalizadas a repetición en relación a picaduras de himenópteros, lo que es excepcional. Es importante destacar que los niños tienen menos riesgo que los adultos de presentar reacciones severas ante nuevas exposiciones $^{3}$. En nuestra serie, la mitad de los pacientes eran niños, y todos comenzaron su IT después de los 5 años.

Para prevenir reacciones de induración locales, pueden utilizarse antihistamínicos e incluso, dosis bajas de corticoides orales el día de la IT y el día previo. También es útil disminuir el volumen por inyección separando la dosis de

\section{REFERENCIAS}

1. Bousquet J, Lockey R, Maluing H. Allergen immunotherapy: therapeutic vaccines for allergic diseases. WHO Position Paper. Allergy 1998; 53 (Suppl. 54): 2-42.

2. Antonicelli L. Epidemiology of Hymenoptera venom allergy. En: Bonifazi F, Bilo MB, Antonicelli $\mathrm{L}$, editores. Insect Allergy. Up to date 2000. Nápoles, JGC s.r.l. Editions, 2002; 11-26.

3. Bonifazi F, Jutel M, Biló B, Birnbaum J, Muller U, and the EAACI Interest Group on Insect Venom Hypersensitivity. Prevention and treatment of mantención y poniendo la mitad en cada brazo, modalidad que hemos utilizado en todos nuestros pacientes.

Luego de una reacción anafiláctica por picadura de himenópteros, el riesgo de presentar otra reacción severa ante una nueva picadura es de 57 a $75 \% 0^{3}$. Los pacientes que han presentado una reacción alérgica generalizada por picadura de abeja tienen mayor riesgo de presentar una reacción severa ante una nueva picadura, en comparación con quienes han sido picados por avispas $^{3,8,10}$. La IT permite disminuír la frecuencia y severidad de estas reacciones en cifras importantes: en estudios prospectivos no controlados con pruebas de provocación (nuevas picaduras), durante el curso de la IT, solamente 0 a $9 \%$ de los pacientes alérgicos a véspidos y $20 \%$ de los pacientes alérgicos a abeja presentaron nuevas reacciones ${ }^{3}$.

La Academia Europea de Alergología e Inmunología Clínica (EAACI), señala un mejor pronóstico cuando la IT se mantiene al menos 5 años ${ }^{13,15-17}$, pudiendo plantearse espaciar la dosis mensual a intervalos de 6 semanas, incluso desde el segundo año de $\mathrm{IT}^{17}$, lo que mejora la adherencia y disminuye los costos. Es el caso de los paciente $\mathrm{n}^{\circ} 1 \mathrm{y} \mathrm{n}^{\circ} 2$, quienes completaron 3 años de dosis mensuales iniciando luego otros 2 años de dosis cada 6 semanas. Sería muy importante contar a futuro con centros especializados a lo largo del país, ya que la mayoría de los consultantes proviene de regiones alejadas y en el caso de nuevos pacientes, es posible que ello impida la realización de este tratamiento.

hymenoptera venom allergy: guidelines for clinical practice. Allergy 2005; 60: 1459-70.

4. King TP, Guralnick M. Hymenoptera allergens. En: Lockey R, Bukantz S. Allergens and Allergen Immunotherapy. Second Edition, Revised and Expanded. New York. Ed. Marcel Dekker 1999; 15568.

5. Simons FE, Gu X, Simons JK. Adrenaline absortion in adults: intramuscular versus subcutaneous injection. J Allergy Clin Immunol 2001; 108: 871-3.

6. Sampson Ha, Muñoz-Furlong A, Campbell RL, ADkinson Nf Jr, Bock Sa, Branum A, et al. Second symposium on the definition and management of 
anaphylaxis: summary report. Second National Institute of Allergy and Infectious Disease/Food Allergy and Anaphylaxis Network symposium. $J$ Allergy Clin Immunol 2006; 117: 391-7.

7. MuelLer UR. Insect sting allergy: clinical picture, diagnosis and treatment. New York: Gustav Fischer Verlag; 1990.

8. Muller U, Mosbech H. Position paper. Immunotherapy with Hymenoptera venoms. EAACI. Allergy 1993; 48 (Suppl 14): 37-46.

9. Fernández J, Soriano V. Inmunoterapia con veneno de himenópteros. Alergol Inmunol Clin 2000; 15: 357-65.

10. Biló BM, Rueff F, Mosbech H, Bonifazi F, OudeElBERINK JNG, and the EAACI interest group on insect venom hypersensitivity. Diagnosis of Hymenoptera venom allergy. Allergy 2005; 60: 1339-49.

11. Nevot S, Guilarte M. Hipersensibilidad a veneno de himenópteros. En Protocolos diagnósticos y terapéuticos en pediatría: Inmunología clínica y alergología [2003] Disponible en http://www.aeped.es/ protocolos/alergia/index.htm. Consultado el 15 de junio de 2006.

12. Laurent J, Smiejan Ja, Bloch-Morot E, Herman D. Safety of hymenoptera venom rush immunotherapy. Allergy 1997; 52: 94-6.

13. Portnoy JM, Moffitt JE, Golden Dbk, Bernstein IL, Berger WE, Dykewicz MS et Al. Stinging insect hypersensitivity: a practice parameter. J Allergy Clin Immunol 1999; 103: 969-80 .

14. Golden D. Insect allergy in children. Curr Opin Allergy Clin Immunol 2006; 6: 289-93.

15. Incorvala C, Pucci S, Pastorello EA. Clinical aspects of Hymenoptera venom allergy. Allergy 1999; 54 (Suppl. 58): 50-2.

16. Armisén C, Vidal C, López-Carballo A, Purriños Mu, Fernández-Ovide E. Alergia a veneno de himenópteros: epidemiología del área sanitaria de Santiago de Compostela. Alergol Inmunol Clin 2001; 16: 213-6.

17. Golden Db, Kwiterovich Ka, Kagey-SobotKa A, Lichtenstein Lm. Discontinuing venom immunotherapy: extended observations. J Allergy Clin Immunol 1998; 101: 298-305. 